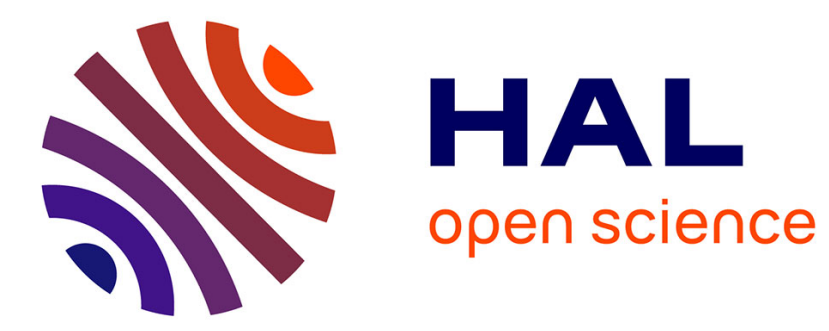

\title{
MILLIMETER-WAVE SPECTROSCOPY OF CESIUM RYDBERG STATES AND POSSIBLE APPLICATIONS TO FREQUENCY METROLOGY
}

\author{
J. Raimond, P. Goy, G. Vitrant, S. Haroche
}

\section{> To cite this version:}

J. Raimond, P. Goy, G. Vitrant, S. Haroche. MILLIMETER-WAVE SPECTROSCOPY OF CESIUM RYDBERG STATES AND POSSIBLE APPLICATIONS TO FREQUENCY METROLOGY. Journal de Physique Colloques, 1981, 42 (C8), pp.C8-37-C8-43. 10.1051/jphyscol:1981805 . jpa-00221700

HAL Id: jpa-00221700 https://hal.science/jpa-00221700

Submitted on 1 Jan 1981

HAL is a multi-disciplinary open access archive for the deposit and dissemination of scientific research documents, whether they are published or not. The documents may come from teaching and research institutions in France or abroad, or from public or private research centers.
L'archive ouverte pluridisciplinaire HAL, est destinée au dépôt et à la diffusion de documents scientifiques de niveau recherche, publiés ou non, émanant des établissements d'enseignement et de recherche français ou étrangers, des laboratoires publics ou privés. 


\title{
MILLIMETER-WAVE SPECTROSCOPY OF CESIUM RYDBERG STATES AND POSSIBLE APPLICATIONS TO FREQUENCY METROLOGY
}

\author{
J.M. Raimond, P. Goy, G. Vitrant and S. Haroche \\ Laboratoire de Physique de l'Ecole Normale Supérieure, 24 me Lhomond, \\ 75231 Paris Cedex 05, France
}

\begin{abstract}
An extensive high-resolution study of the millimeter-wave spectrum of Cesium Rydberg states is presented, yielding new precise values of the ns, $n P, n D$ and $n F$ states quantum defects $(23 \leqslant n \leqslant 45)$, and of their fine and hyperfine structures. The quantum defects values allow us to predict the frequency of any $n, l \rightarrow n^{\prime}, l^{\prime}(n \geqslant 20, l \leqslant 3)$ transition, with a MHz accuracy. This precise mapping of the Cesium Rydberg states can provide a new secondary frequency standard in the $50 \mathrm{GHz}-10 \mathrm{THz}$ range. Possible interesting metrological applications are discussed.
\end{abstract}

Introduction - This paper presents an extensive study of the spectroscopy of cesium Rydberg states. The energy spectxum of alkalis Rydberg states is similar, in many ways, to the hydrogenic one. The energy of these states may be expressed as $\mathrm{E}=-\mathrm{R} /(\mathrm{n}-\varepsilon)^{2}$, where $\mathrm{R}$ is the Rydberg constant relative to the nucleus mass, $n$ the principal quantum number, and $\varepsilon$ the quantum defect which reflects the core perturbation experienced by the outer electron. $\varepsilon$ depends mostly on the angular momentum, decreasing with increasing $\ell$. $\varepsilon$ exhibits also a slight dependence on $J$, reflecting the fine structure coupling, and a very slow variation with $n$, which can be put in evidence only in very precise experiments.

We present here the most precise determination of $\varepsilon$ for $S, P, D$ and $F$ states $(23 \leqslant n \leqslant 45)$. We give also new values for the fine structures of these states, and report the first measurement, to our knowledge, of hyperfine structures in Rydberg states ( $S$ and $P_{1 / 2}, 23 \leqslant n \leqslant 28$ ). The quantum defects values given here allow one to predict the position of any $n, \ell(n \geqslant 20, \ell \leqslant 3)$ level, with a MHz accuracy. This mapping of the Cesium Rydberg states could provide a new secondary frequency standard grid in the microwave range, from a few $\mathrm{GHz}$ to $10 \mathrm{THz}$. Other possible applications to metrology are discussed.

The principle of the experiment is similar to the one of previous works on Sodium Rydberg states spectroscopy (1) (2) (3) : the Rydberg atoms are prepared by a pulsed laser excitation, and then interact during a few microseconds with a frequency stabilized tunable millimeter-wave field, which induces transitions between Rydberg levels.

\section{Experimental set-up}

The Rydberg states are produced in a Cesium atomic beam by a stepwise laser excitation. Two pulsed dye lasers, pumped by the same Nd : YAG (third harmonic) laser, induce the transitions $6 \mathrm{~S}_{\frac{1}{2}} \rightarrow 6 \mathrm{P}_{3 / 2}\left(8521 \AA\right.$ ) and $6 \mathrm{P}_{3 / 2} \rightarrow \mathrm{nS}$ or $\mathrm{nD}$ (around $5100 \AA$ for $20 \leqslant n \leqslant 50$ ). The Rydberg states interact then with the microwave during a time $\mathrm{T}_{0}$, varying from 1 to $10 \mathrm{\mu S}$, and are detected by the well-know Field-Ionization 
Technique (1) : after the delay $\mathrm{T}_{0}$, an electric field ramp is applied to the atoms, raising to $1 \mathrm{kV} / \mathrm{cm}$ in about 1 s. Different levels ionize in different fields, i.e. at different times. The produced electrons are detected and amplified in an electron multiplier. The time-resolved signals corresponding to the initial and final states of the microwave transition are averaged over a few tens of laser shots in a Boxcar integrator, and recorded as a function of the microwave frequency.

The microwave field is produced by direct sources or by harmonic generation. Direct sources are Back-Wave Oscillators (Thomson-CSF Carcinotrons). We used a set of 9 BWo's covering partly the $50-480 \mathrm{GHz}$ range (each of them can be voltage-tuned over a range of about 10 of its nominal frequency). In order to measure precisely the microwave frequency, we use the harmonic mixing with an X-Band Klystron ( 8.1 $12.4 \mathrm{GHz}$ ), which is stabilized by reference to a quartz oscillator (Mos-5 microwave system). Its frequency is measured, with a $10^{-9}$ accuracy, in a $12 \mathrm{GHz}$ counter, whose reference clock is provided by a long wave receiver for frequency standards (Adret $\left.n^{\circ} 4101 \mathrm{~A}\right)$. The klystron power is sent to a home-made harmonic multiplier, using a packaged schottky diode, placed in a millimeter wave guide (bands V, E, W, F), mixing the BWo signal with the $\mathrm{p}^{\text {th }}$ harmonic of the klystron. The intermediate frequency beat note is amplified and counted, yielding a precise value of the BWo frequency.

Moreover, this signal can be used to lock the BwO frequency. A phase-locking system is used up to $200 \mathrm{GHz}$. A signal proportional to the relative phase of the beat note and of a reference given by a frequency synthesizer is used as a correction voltage, added to the BWo line voltage. The linewidth of the BWo can so be reduced, from the free running value ( $1 \mathrm{MHz}$ ), down to a few $\mathrm{Hz}$. The microwave frequency can be swept by changing the synthesizer one. At higher frequencies (> 200 GHz), the Klystron multiplied phase noise makes this technique less efficient. It is then worth using a frequency locking system : the beat note $(0.1-15 \mathrm{MHz})$ is fed into a frequency-voltage converter, whose output, summed with a tunable offset, is used as a correction voltage. The linewidth of a $460 \mathrm{GHz}$ BWO is reduced from 2 MHz to 1 $\mathrm{kHz}$ only. Some detailed informations about these frequency locking techniques are to be published elsewhere.

Some Rydberg-Rydberg transitions are very sensitive to the microwave field (saturating power of the order of $0.1 \mathrm{nw} / \mathrm{cm}^{2}$ for a $\mathrm{ns}-\mathrm{nP}$ transition), and the huge powers of the BWO's (10 $\mathrm{mW}$ to $5 \mathrm{w}$ ) makes difficult to get rid of power broadening problems. For such sensitive transitions, it is worth using directly the harmonic of the klystron given by the multiplying schottky diode. From a klystron power of 100 $\mathrm{mW}$ at $11.5 \mathrm{GHz}$, one can obtain, up to $0.1 \mathrm{~mW}$ at $80 \mathrm{GHz}, 0.1 \mu \mathrm{W}$ at $230 \mathrm{GHz}$. This power is much easier to control, either by attenuating the klystron, or by modifying the diode bias. AlI single photon $\mathrm{nS} \rightarrow \mathrm{nP}$ transitions up to $340 \mathrm{GHz}$ (microwave power $>1 \mathrm{nW}$, harmonic number $\mathrm{p}=38$ ) can be observed with this cheap and simple arrangement. Moreover, all the frequencies greater than the cut-off of the waveguide used ( $f \geqslant 41 \mathrm{GHz}, \mathrm{p} \geqslant 4$ for $\mathrm{V}$ band) are available, choosing the adequate klystron frequency and harmonic number : the same source is used through the whole spectrum, giving a great flexibility to the experimental apparatus.

\section{Presentation of the results}

We have observed more than 40 lines, involving one phioton $\mathrm{ns}_{1 / 2} \rightarrow \mathrm{nP}_{1 / 2}, 3 / 2$; $\mathrm{nD}_{5 / 2} \rightarrow(n-3) \mathrm{F}_{5 / 2}, 7 / 2$ and $\mathrm{nD}_{5 / 2} \rightarrow n P_{3 / 2}$ transitions, and two photons $n S_{1 / 2} \rightarrow(n+1) S_{1 / 2}$ and $\mathrm{nD}_{5 / 2} \rightarrow(n+1) D_{3 / 2}, 5 / 2$ ones, n ranging from 23 to 45 . For $n s \rightarrow(n+1) s$ transitions, the intermediate level $\mathrm{nP}_{3 / 2}$ is very close to the center of the energy gap (the detuning is only $8 \mathrm{MHz}$ for $\mathrm{n}=42$ ). Hence, the line saturating power is very low (down to $10 \mathrm{nW} / \mathrm{cm}^{2}$ ), and the transitions up to $110 \mathrm{GHz}$ have been observed with the Klystron harmonics. On the other hand, the $\mathrm{nD} \rightarrow(\mathrm{n}+1) \mathrm{D}$ transitions require large amounts of microwave (the intermediate $(n-2) \mathrm{F}$ level is $16 \mathrm{GHz}$ apart from the energy gap center for $n=30$ ), and the Bwo has to be used. 
for single photon ones. The Doppler effect $(100 \mathrm{kHz})$ affects only the narrowest $S-S$ two photon lines : the main limitation to the single photon linewidth seems to be due to stray electric field inhomogeneities $(100 \mathrm{mv} / \mathrm{cm})$, inducing stark shifts on the very sensitive Rydberg states. These shifts are cancelled for two photon transitions, since both levels experience almost the same displacement. We did not perform Doppler-free two photon transitions in the standing wave of a cavity, as in previous experiments on Sodium (2) : Cesium atoms are much slower, and the time of flight across the cavity is much longer than the Rydberg states lifetime.

Fig. 1 shows a recording of the $28 \mathrm{~S}_{1 / 2} \rightarrow 28 \mathrm{P}_{1 / 2}$ transition. The spectrum exhibits splittings due to the hyperfine interaction with the $I=7 / 2$ nuclear spin. The assignment of the four hyperfine lines is displayed in the insert. The splitting of the $28 \mathrm{~S}$ level $(4.7 \pm 0.8 \mathrm{MHz})$ is clearly resolved. The splitting of the $\mathrm{P}_{1 / 2}$ level $(1.1 \pm 0.2 \mathrm{MHz})$ is of the order of the linewidth, and barely obsexvable. Another hyperfine splitting is appearent in fig. 2, showing a record of the two photon $36 S_{1 / 2} \rightarrow 37 S_{1 / 2}$ line. One observes two components, corresponding to the two $\Delta F=0$ transitions, which are the only ones allowed. Hence, the splitting (70 $\mathrm{kHz}$ ) is only the difference of the hyperfine structures of the two $S$ levels. The $P_{3 / 2}, D$ and $F$ hyperfine structures, smaller and more complex, are not resolved in our experiments.

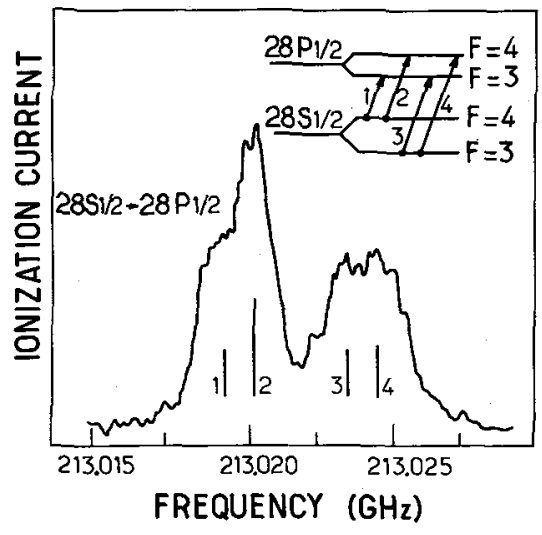

Fig. 1 : Record of the $28 S_{1 / 2} \rightarrow 28 P_{1 / 2}$ transition. The insert displays the assignment of the four hyperfine components of the line.

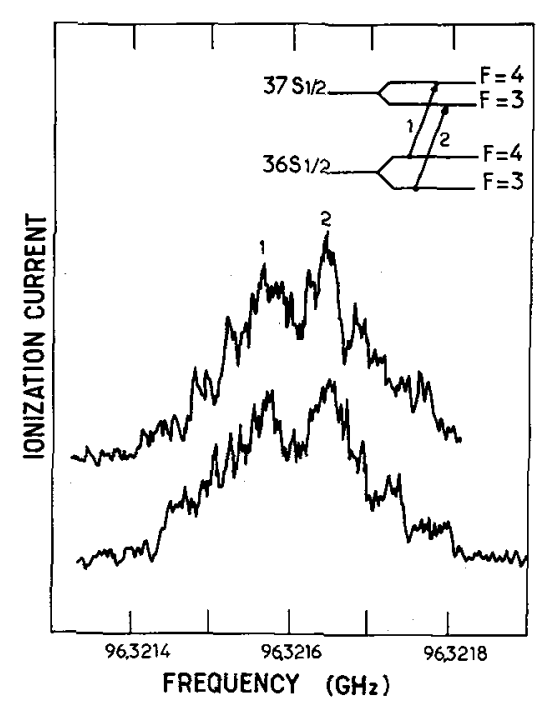

Fig. 2 : Record of the $36 S_{1 / 2} \rightarrow 37 S_{1 / 2}$ two photon transition. The insert displays the assignment of the hyperfine components of the line.

We have measured the hyperfine structures of $23,25,26$ and $28 \mathrm{~S}_{1 / 2}$ and $\mathrm{P}_{1 / 2}$ levels, which turn out to be respectively : $9.2 \pm 0.8 ; 5.8 \pm 0.8 ; 5.7 \pm 0.8$; $4.7 \pm 0.8 \mathrm{MHz}$ for $\mathrm{S}_{1 / 2}$ levels and $2.23 \pm 0.2 ; 1.6 \pm 0.2 ; 1.25 \pm 0.2 ; 1.1 \pm 0.2 \mathrm{MHz}$ for $P_{1 / 2}$ ones. This observation is, to our knowledge, the first determination of hyperfine structures in Rydberg states, with $n \simeq 30$.

The fine structures of $P, D$ and $F$ levels are much more easily resolved. The $P$ 
fine structures have been measured, on $s_{1 / 2} \rightarrow P_{1 / 2}, \frac{3}{2}$ lines in a wide range of $n$ values (23 to 42), with a precision of about $1 \mathrm{MHz}$. The F fine structures, which are known to be inverted from (4), have been measured for three levels on $D_{5 / 2} \rightarrow F_{5} / 2,7 / 2$ transitions. The $\mathrm{D}$ one is more difficult to observe on one photon lines, since the laser excites almost only the $D_{5 / 2}$ level. Only one structure has been observed, on the two photon $31 D_{5 / 2} \rightarrow 32 D_{5 / 2}, 3 / 2$ transition.

Analysis of the results : the quantum defects formulae

Our results give new precise values of the $S, P, D$ and $F$ quantum defects. The frequency of the nlJ $\rightarrow$ ' ' ' J' transition can be expressed as :

$$
F=R\left[\left(n-\varepsilon_{\ell J}(n)\right)^{-2}-\left(n^{\prime}-\varepsilon_{\ell^{\prime} J^{\prime}}\left(n^{\prime}\right)\right)^{-2}\right]
$$

where $R$ is the Rydberg constant for Cesium ( $R=3,289828299 \quad$ (20) $10^{9}$ MFiz). The quantum defect $\varepsilon_{\ell J}$ depends mainly on $\ell$ and $J$ (the $J$ dependence reflects the fine structure coupling). The $n$ dependence of $\varepsilon_{l J}$ can be expressed by the Ritz formula, limited to two terms for $n \geqslant 20$ :

$$
\varepsilon_{\ell J}(n)=\varepsilon_{\ell J}{ }^{(\infty)}+a_{\ell J}\left[n-\varepsilon_{\ell J}{ }^{(\infty)}\right]^{2}
$$

All the $\varepsilon_{\ell J}(\infty)$ and $a_{\ell J}$ coefficients $(\ell \leqslant 3)$ can be deduced from our results.

The frequency of a $n S \rightarrow(n+1)$ s transition yields a "mean" quantum defect of the two $S$ states, $\varepsilon^{\star}(n)$. Extrapolating $\varepsilon^{\star}$ to $n=\infty$, we get the value of $\varepsilon_{0,1 / 2}(\infty)$, then of $a_{0,1 / 2}$. The same calculation applied to the $n D_{5 / 2} \rightarrow(n+1) D_{5 / 2}$ lines gives the values of $\varepsilon_{2,5 / 2}{ }^{(\infty)}$ and $\mathrm{a}_{2,5 / 2}$. The $\varepsilon$ and a values for $\mathrm{P}$ and $\mathrm{F}$ levels are then calculated from the one photon transitions frequencies. The results are summarized in table 1. The calculated variations of the quantum defects for $S, P, D$ and $F$ states

Table 1

\begin{tabular}{l|l|l}
\multicolumn{1}{|c}{$\varepsilon_{\ell J}{ }^{(\infty)}$} & $a_{\ell J}$ \\
$\mathrm{~S}_{1 / 2}$ & $4.049325 \pm 1.510^{-5}$ & $0.2462 \pm 510^{-3}$ \\
$\mathrm{P}_{1 / 2}$ & $3.591556 \pm 310^{-5}$ & $0.3714 \pm 410^{-3}$ \\
$\mathrm{P}_{3 / 2}$ & $3.559058 \pm 310^{-5}$ & $0.374 \pm 410^{-3}$ \\
$\mathrm{D}_{3 / 2}$ & $2.475365 \pm 210^{-5}$ & $0.0554 \pm 610^{-3}$ \\
$\mathrm{D}_{5 / 2}$ & $2.466210 \pm 1.510^{-5}$ & $0.067 \pm 510^{-3}$ \\
$\mathrm{~F}_{5 / 2}$ & $0.033392 \pm 310^{-5}$ & $-0.191 \pm 310^{-2}$ \\
$\mathrm{~F}_{7 / 2}$ & $0.033537 \pm 2.510^{-5}$ & $-0.191 \pm 210^{-2}$
\end{tabular}

are shown in fig. $3,4,5,6$ respectively (on fig. 3 and $5, \varepsilon^{\star}(n)$ is also plotted as a dashed line). The only D fine structure measured here doesn't allow us to get directly the $\varepsilon_{2,3 / 2}$ and $a_{2,3 / 2}$ coefficients. Nevertheless, precise values can be obtained by deducing the binding energy of $D_{3 / 2}$ from the $D_{5 / 2}$ measured one, and from optical measurements of $\mathrm{D}$ fine structures (5). The corresponding values of the quantum defect are plotted as triangles in fig. 5, and are remarkably well aligned with our one.

This quantum defect determination can be compared to the best results of optical experiments (5) (6). The corresponding values of $\varepsilon$ are plotted as dotted lines in fig. 3, 4, 5, 6, with the typical errors bars. The agreement is generally good, except for the D levels. The accuracy of our values is typically one order of magni- 


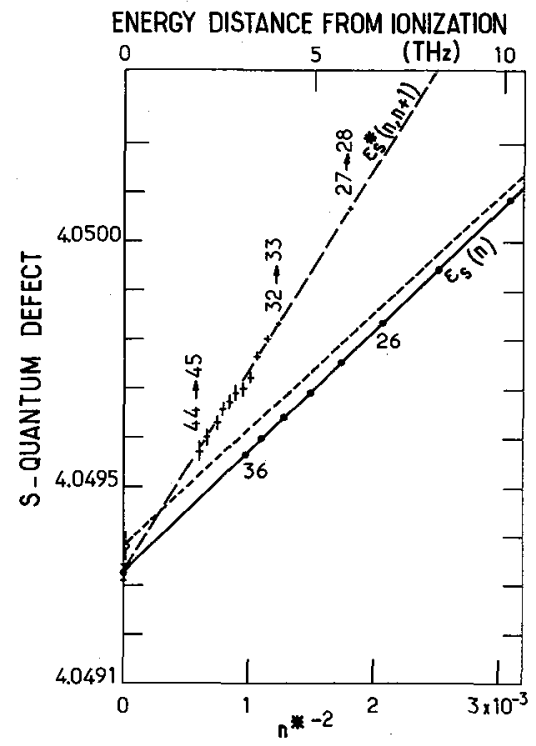

Fig. 3 : Solid line : Variation of $\frac{1}{\varepsilon_{0,1 / 2}}$. Dashed line : variation of the mean quantum defect $\varepsilon^{\star}(n)$, with the experimental values. Dotted line : values deduced from ref. (5) (6).

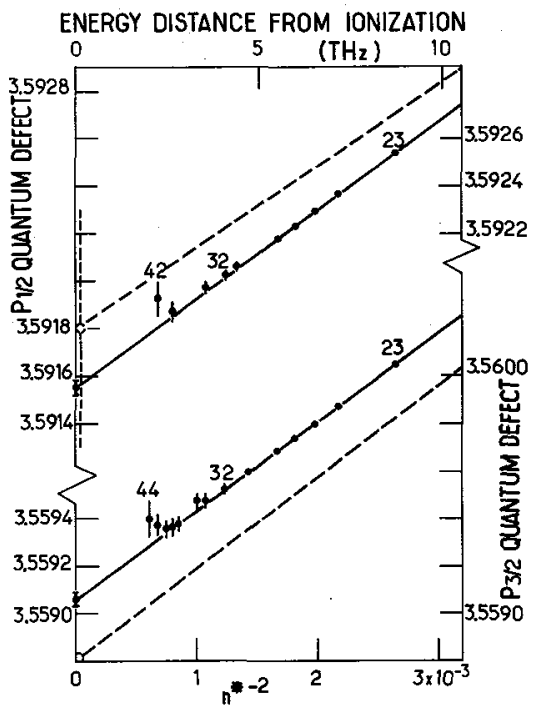

Fig. 4 : Variation of $P_{1 / 2}$ and $P_{3 / 2}$ quantum defect, with experimental values. Dotted line from (5) and (6).

tude better. The values of the quantum defects calculated here allow us to predict the frequency of any $n l-n^{\prime} \ell$ ' transition for $n \geqslant 20, \ell \leqslant 3$. The agreement between observed and calculated values is remarkably good (except for the upper $P$ and $F$ levels, very sensitive to Stark shifts) : the typical discrepancy is of the order of $1 \mathrm{MHz}$ for one photon lines, and only a few tens of $\mathrm{kHz}$ for two photon ones.

Another interesting feature of this determination is that it is independent of the ionization limit value. Moreover, calculating from eq. (1) the binding energy of a level, and knowing from (5) its distance to the ground state, we can get a new value for the ionization limit of cesium. Analyzing in such a way 13 different ns levels, and $21 \mathrm{nD}_{5 / 2}$ ones, we get:

$$
\mathrm{E}_{I}=31406.4676(33) \mathrm{cm}^{-1}
$$

(the quoted error is the standard deviation of the results). This value agrees well with that given in (5), with a slightly smaller uncertainty.

The fine structure intervals can be expressed as a serie of odd powers of $n-\varepsilon_{\ell, J, J+1}(n)$, where $\varepsilon_{\ell, J, J+1}(n)$ is the average of the two fine structure levels quantum defects :

$$
\begin{array}{r}
\Delta f_{S}(n \ell J+n \ell J+1)=A\left[n-\varepsilon_{\ell, J, J+1}(n)\right]^{-3}+B\left[n-\varepsilon_{\ell, J, J+1}(n)\right]^{-5} \\
+C\left[n-\varepsilon_{\ell, J, J+1}(n)\right]^{-7}
\end{array}
$$

Our experiments in high lying levels gives only the A coefficient, but the connection with the fine structure values in lower levels allow us to give the values of $\mathrm{B}$ and $\mathrm{C}$ coefficients listed in table 2 . The agreement between the predictions of 


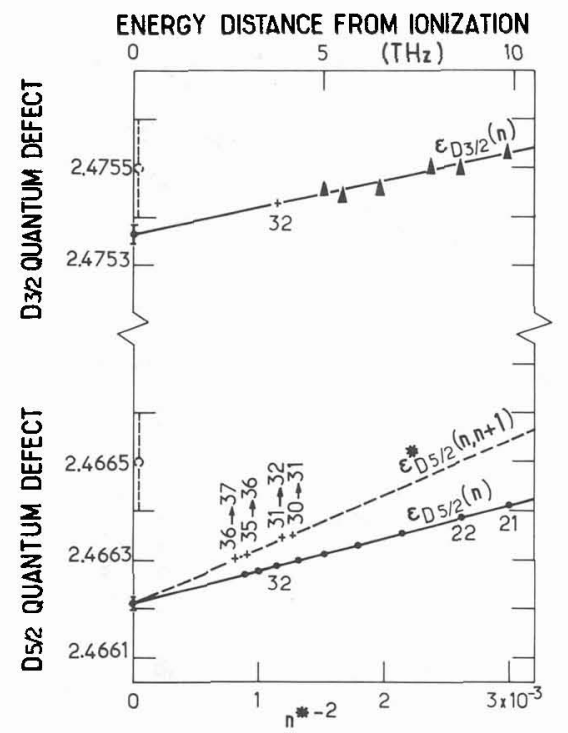

Fig. 5 : Solid lines : our values. Dashed iine : variation of $\varepsilon \star(n)$, and experimental points. Triangles : values of $\varepsilon_{2,3 / 2}$ deduced from $\varepsilon_{2,5 / 2}$ and ref. (5). Dotted points : values deduced from ref. $(5)(6)$. The slope was 0 to the degree of precision of those experiments.

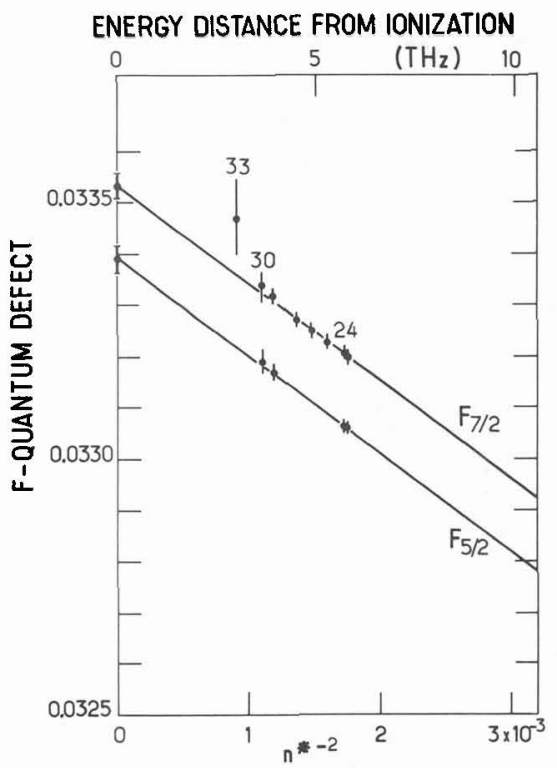

Fig. 6 : Variation of $F_{5 / 2}$ and $F_{7 / 2}$ quantum defects.

eq. 3 and the observed values is excellent wholeover the energy spectrum : the typical discrepancy is only of the order of $0.1 \%$.

Table 2

A $(\mathrm{MHz})$

$$
\begin{aligned}
& \mathrm{nP}_{1 / 2} \rightarrow \mathrm{nP}_{3 / 2} \\
& \mathrm{nD}_{3 / 2} \rightarrow \mathrm{nD}_{5 / 2} \\
& \mathrm{nF}_{5 / 2} \rightarrow \mathrm{nF}_{7 / 2}
\end{aligned}
$$

$\star$ from ref. (4)
B $(\mathrm{MHz})$

$$
\begin{array}{r}
-5.6(4) 10^{7} \\
-5.8(8) 10^{7} \\
1.22210^{7}
\end{array}
$$

5 (
C $(\mathrm{MHz})$
3.9 (1) $10^{8}$

0

$-3.37610^{7}$

\section{Conclusion}

The experiments presented here can prepare the way to metrological applications of the Rydbexg states. The remarkable agreement between the line frequencies calculated from our quantum defects values, and the observed ones, makes us confident that the frequency of any $n \ell \rightarrow n \cdot l \cdot(n \geqslant 20, \ell \leqslant 3)$ transition can be calculated with a MHz accuracy, reduced to a few tens of $\mathrm{kHz}$ for $\mathrm{S}-\mathrm{S}$ transitions. In fact, this uncertainty could be reduced to $1 \mathrm{kHz}$ by a careful cancellation of stray electric fields and Doppler effect. This could provide a new frequency reference grid in the $50 \mathrm{GHz}$ - $10 \mathrm{THz}$ range (the $20 \mathrm{~S}$ level is $12.9 \mathrm{THz}$ apart from the ionization limit). It could be possible, in such a way, to compare indirectly and easily, but with a good accuracy, the frequency of a far infrared source to a frequency standard. 
Another interesting extension of this experiment could yield a new determination of the light velocity. Lee et al. (7), stoicheff et al. (8), have measured, with a great precision, the position of the Rubidium Rydberg levels, with respect to the ground state, in units of wavelength. A microwave spectroscopic experiment on Rubidium atoms should give the relative positions of the same levels, in units of frequency. A new value of $c$ could be deduced from both these experiments. One should quote, however, that the precision of both optical and microwave experiments should be increased to give a more precise value than the one available now.

These studies of alkalis Rydberg states prepare also the way to the microwave spectroscopy of Hydrogen and Deuterium Rydberg states. The appiications of some techniques already used in other types of experiments (cooled Hydrogen beams, stray electric field suppression) should lead to a precise determination of the Rydberg constant in units of frequency, and of the neutron to proton mass ratio.

\section{References}

(1) FABRE C., HAROCHE S. and GOY P., Phys. Rev. A 18, (1978), 229

(2) GOY P., FABRE C., GROSS M. and HAROCHE S., J. Phys. B : Atom. Molec. Phys. $13,(1980), \mathrm{L}-83$

(3) FABRE C., HAROCHE S. and GOY P., Phys. Rev. A 22, (1980), 778

(4) FREDRIKSSON K., LUNDBERG H. and SVANBERG S., Phys. Rev. A $21,(1980), 241$

(5) LORENZEN C.J., WEBER K.H. and NIEMAX K., Optics Comm. 33, $(1980), 271$

(6) LORENZEN C.J. and NIEMAX K., J. Quant. Spectr. Radiat. Transfer 22, (1979) 247

(7) LEE S.A., HELMKE J. and HALI J.L., Optics Letters 3, (1978), 141

(8) STOICHEFF B.P. and WEINBERGER E., Laser spectroscopy IV, walter $\mathrm{F}$. and Rothe K.W., ed. Springer Verlag (1979) 